\title{
Comparison between Fasting and Non-Fasting Sample for the Determination of Serum Lipid Profile
}

\author{
Shivangi Ghildiyal ${ }^{1}$, Ashish Prakash Anjankar ${ }^{2}$, Prakash Keshaorao Kute ${ }^{3}$
}

${ }^{1}$ Department of Biochemistry, Jawaharlal Nehru Medical College, Sawangi, Meghe, Wardha, Maharashtra, India. 2Department of Biochemistry, Jawaharlal Nehru Medical College, Sawangi, Meghe, Wardha, Maharashtra, India. ${ }^{3}$ Department of Biochemistry, Jawaharlal Nehru Medical College, Sawangi, Meghe, Wardha, Maharashtra, India.

\section{ABSTRACT}

\section{BACKGROUND}

For determination of serum lipid profile, traditionally we use fasting blood sample. Though it has been the most reliable method for testing lipid profile, it has some drawbacks. Fasting is not easy for some people specially children, diabetics and also it is a barrier for population screening. So, intent of our study is to check the authenticity of results obtained using non-fasting samples by correlating it with the results obtained using fasting samples. We wanted to estimate \& compare fasting and non-fasting lipid profile values in severe diabetic patients and in apparently healthy controls.

\section{METHODS}

This analytical cross sectional study included 40 apparently healthy controls and 40 diabetic patients as participants confirmed by history and biochemical tests. Blood sample was collected from each patient two times; once after 10-12 hours fasting and other as a random sample. Lipid profile parameters were estimated using standard tests. Statistical analysis was done by using Pearson's correlation coefficient. Data analysis was carried by Statistical Package SPSS and Microsoft Excel and $p<0.05$ was considered as level of significance.

\section{RESULTS}

In apparently healthy controls differences between fasting and non-fasting concentrations were small and clinically insignificant for lipid profile parameters like total cholesterol $(\mathrm{p}=0.861)$, LDL-cholesterol $(\mathrm{p}=0.203)$ and HDL-cholesterol $(p=0.916)$. The difference was statistically significant $(p=0.001)$ for triglycerides.

\section{CONCLUSIONS}

Fasting samples are preferable for serum lipid profile measurement in all individuals with serum triglyceride levels greater than $350 \mathrm{mg} / \mathrm{dL}$. But, non-fasting samples for lipid profile can be used for cardiovascular risk determination in the general people as it reduces patient's inconvenience and promotes patient acquiescence towards lipid profile checking.

\section{KEY WORDS}

Fasting, Non-Fasting, Lipid Profile, Cardiovascular Disease
Corresponding Author:

Dr. Ashish Prakash Anjankar,

Department of Biochemistry,

Jawaharlal Nehru Medical College,

Sawangi, Meghe, Wardha-442001,

Maharashtra, India.

E-mail: ashish.anjankar@gmail.com

DOI: $10.14260 /$ jemds/2020/243

Financial or Other Competing Interests: None.

How to Cite This Article: Ghildiyal S, Anjankar AP, Kute PK. Comparison between fasting and nonfasting sample for the determination of serum lipid profile. J. Evolution Med. Dent. Sci. 2020;9(14):1122-1125, DOI: $10.14260 /$ jemds/2020/243

Submission 29-01-2019,

Peer Review 15-03-2020,

Acceptance 21-03-2020,

Published 06-04-2020. 


\section{BACKGROUND}

We routinely perform serum lipid profile test for cardiovascular disease (CVD) risk prediction. ${ }^{1}$ Lipid profile test is routinely done in fasting blood specimen. It includes four basic parameters: total cholesterol (TC), triglycerides (TG), high density lipoprotein cholesterol (HDL-C) and low density lipoprotein cholesterol (LDL-C). Fasting means overnight 12-14 hours dietary constraint with the exclusion of water and medicines. Fasting sample is favoured for lipid profile due to two important reasoning: (a) post prandial (after a meal) triglycerides remain inflated for several hours, ${ }^{2}$ (b) most reference values for serum lipids are fixed on fasting blood specimen. National Cholesterol Education Program (NCEP) ${ }^{3}$ and European guidelines ${ }^{4}$ also endorse measuring lipid profile in fasting blood specimen for appraisal of CVD risk. Interestingly, while assessing lipid profile for CVD risk appraisal, superiority of fasting specimen over non-fasting specimen has not proven by ancillary evidence. An online report in Archives of Internal Medicine (November 12) found that there is only marginal difference in lipid levels when measured in a fasting or non-fasting state. ${ }^{5}$ NCEP guidelines also granted total cholesterol (TC) and HDL-C in non-fasting specimen as these lipid parameters are nearly similar in fasting and non-fasting state.6,7 Adult treatment panel III recommended non- HDL-C (TC-HDL-C) may also be measured in the non-fasting state. Eberly et al. (2003), ${ }^{8}$ Bansal et al. (2007), ${ }^{9}$ and Nordestgaard et al. (2007) ${ }^{10}$ have advised that levels of non-fasting triglycerides may better or similarly anticipate CVD events than levels of fasting triglycerides.

Nevertheless, in routine clinical practice the lipid profile is generally measured after 12-14 hours of fasting. Though it has been the most reliable method for testing lipid profile, it has some drawbacks. Fasting is not easy for some people especially children and diabetics. Lipid profile in fasting state acts as a barrier for population screening. Also for patients, physicians and testing laboratories, it would be more convenient and efficient to measure lipid levels in a nonfasting setting. ${ }^{11-15}$ So, unless there's a really good reason for fasting samples to be tested, non-fasting sampling is much more convenient for all, from a practical perspective. So, intent of our study is to check the authenticity of results obtained using non-fasting samples by correlating it with the results obtained using fasting samples.

We wanted to estimate \& compare fasting and non-fasting lipid profile values in severe diabetic patients and in apparently healthy controls.

\section{METHODS}

It is an analytical cross-sectional study carried out in Department of Biochemistry, of a tertiary care hospital, among 80 patients of diabetes mellitus (de-novo, follow cases of diabetes mellitus, patients with complications of diabetes mellitus). The sample size was determined using Krejcie and Morgan methodology of calculation of sample size-

$$
N=\frac{X^{2 *} N^{*} P^{*}(1-\mathrm{P})}{\left(\mathrm{M} E^{2 *}(N-1)\right)+\left(X^{2 *} P^{*}(1-P)\right)}
$$

Where:

$\mathrm{N}=$ sample size

$\mathrm{X}^{2}=$ Chi-square for the specified confidence level at 1 degree of freedom

$\mathrm{N}=$ Population Size

$\mathrm{P}=$ Population Proportion (.50 in this table)

$\mathrm{ME}=$ Desired Margin of Error (expressed as a proportion)

It included 40 apparently healthy controls and 40 diabetic patients (cases) who were confirmed by history and biochemical tests. All diabetic subjects had chronic HbA1c greater than 8.0, a clinical diagnosis of mild or moderate NPDR, and no other retinal disease. The control subjects did not have diabetes or other retinal disease. Written consent was gathered from each subject. Institutional Ethical Committee (IEC) has affirmed this study protocol.

\section{Inclusion Criteria}

Diagnosed cases of type 2 diabetes mellitus of all age groups and of either sex.

\section{Exclusion Criteria}

- Hypo-functioning of thyroid gland.

- Chronic renal failure, Nephrotic syndrome.

- Patients on corticosteroids or oral contraceptives.

- $\quad$ Patients on lipid lowering drugs.

Blood sample was collected from each patient two times: once after 12-14 hours fasting and other as a random sample. All the blood samples were collected into plain bulbs. Lipid profile includes measurements of TC, TG, LDL-C and HDL-C. Analysis of lipid profile was done on Randox Biochemistry Fully automated autoanalyser.

\begin{tabular}{|ccc|}
\hline No. & Parameter & Method \\
1. & Total Cholesterol & $\begin{array}{c}\text { Enzymatic method - Cholesterol esterase, cholesterol } \\
\text { oxidase and peroxidase } \\
\text { Direct Enzymatic method }\end{array}$ \\
2. & HDL-C & $\begin{array}{c}\text { Enzymatic method- Liquid stable Glycerol phosphate } \\
\text { oxidase and peroxidase; End point }\end{array}$ \\
3. & Triglycerides & Indirect method- Friedwald Equation 16 VLDL-C $=$ \\
4. & VLDL-C and LDL-C & TG LDL-C = TC - HDL-C - (TG/5). \\
\hline \multicolumn{2}{|c|}{ Table I. Lipid Profile Parameters with Their Method of Estimation } \\
\hline
\end{tabular}

\section{Statistical Analysis}

Data was entered in Microsoft Excel. Statistical analysis was done by using Pearson's correlation coefficient. Data analysis was carried by Statistical Package SPSS and Microsoft Excel and $\mathrm{p}<0.05$ is considered as level of significance.

\section{RESULTS}

\begin{tabular}{|cccc|}
\hline Parameter & Fasting & Non-Fasting & p Value \\
TC & $174.33( \pm 20.86)$ & $172.26( \pm 20.95)$ & 0.861 \\
TG & $147.83( \pm 15.04)$ & $178.20( \pm 16.84)$ & $0.001 \mathrm{~S}$ \\
LDL-C & $131.77( \pm 9.83)$ & $129.65( \pm 8.63)$ & 0.203 \\
HDL-C & $41.96( \pm 5.87)$ & $42.17( \pm 5.24)$ & 0.916 \\
\hline \multicolumn{4}{|c|}{ Table 2. Serum Lipid Profile in Fasting and Non-Fasting Sample } \\
in Apparently Healthy Controls \\
\hline S-Significance, $\mathrm{p}<0.05$
\end{tabular}


From table 2, in apparently healthy controls differences between fasting and non-fasting concentrations were small and clinically insignificant for lipid profile parameters like total cholesterol $(p=0.861)$, LDL-cholesterol $(p=0.203)$ and HDL-cholesterol $(p=0.916)$. The difference was statistically significant $(\mathrm{p}=0.001)$ for triglycerides.

\begin{tabular}{|cccc|}
\hline Parameter & Fasting & Non-Fasting & p value \\
TC & $269.24( \pm 22.33)$ & $266.54( \pm 24.75)$ & 0.804 \\
TG & $230.53( \pm 18.99)$ & $281.92( \pm 24.88)$ & $0.001 \mathrm{~S}$ \\
LDL-C & $188.44( \pm 10.32)$ & $185.39( \pm 9.93)$ & 0.189 \\
HDL-C & $38.01( \pm 5.11)$ & $36.38( \pm 4.68)$ & 0.901 \\
\hline \multicolumn{4}{|c|}{ Table 3. Serum Lipid Profile in Fasting and Non-Fasting Sample } \\
\multicolumn{4}{|c}{ in Severe Diabetic Patients } \\
\hline \multicolumn{3}{|c|}{ S-Significance, $\mathrm{p}<0.05$} &
\end{tabular}

From table 3, in severe diabetic patients differences between fasting and non-fasting concentrations were small and clinically insignificant for lipid profile parameters like total cholesterol $(p=0.804)$, LDL-cholesterol $(p=0.189)$ and HDL-cholesterol $(p=0.901)$. The difference was statistically significant $(\mathrm{p}=0.001)$ for triglycerides.

\section{DISCUSSION}

TC and LDL-C levels were slightly reduced in non-fasting as compared to fasting specimen. The possible cause for small reduction in TC \& LDL-C levels in non-fasting specimen is most likely haemodilution following fluid intake in association to the meal. ${ }^{17}$ Triglycerides increased and HDL-C decreased in non-fasting as compared to fasting specimen. These changes are mostly possible due to food intake rather than fluid intake. Although triglyceride increase are owed directly to fat intake, the parallel reduction in HDL cholesterol is possibly due to bidirectional lipid exchange between triglyceride-rich lipoproteins and HDL particles. ${ }^{18}$

Our study results corresponded well with the studies carried out by Langsted et al. (2008) ${ }^{11}$, Mora et al. (2008) ${ }^{12}$, Steiner et al. (2011) ${ }^{19}$, Langsted and Nordestgaard (2011) ${ }^{20}$ Sidhu and Naugler (2012).21 These large-scale, populationbased studies have now proven that there is only a slight change in lipids and lipoproteins levels in response to habitual food intake. Among all other studies comparing nonfasting with fasting lipid profiles, minor increase in plasma triglycerides and minor decreases in TC and LDL-C concentrations were noticed, with nominal change in HDL-C concentrations. These minor changes in lipid profile parameters appear to be clinically unimportant.

Since 2009, International guidelines are recommending non-fasting lipid profiles. Several societies' guidelines in the United Kingdom, Europe, Denmark, United States, Canada and Brazil also advocate non-fasting lipid profiles. In 2009, the Danish Society for Clinical Biochemistry endorsed that all laboratories in Denmark should use random non-fasting specimen for lipid profile measurements rather than fasting specimen while offering physicians the option of remeasurement of triglyceride concentrations in the fasting state if non-fasting values aremore than $350 \mathrm{mg} / \mathrm{dL} .{ }^{20,22}$ Since 2009, non-fasting lipid testing has become a clinical benchmark in Denmark and practically after 2015 all laboratories in Denmark have adopted non-fasting lipid profiles.
Sidhu and Naugler (2012) ${ }^{21}$ expressed that fasting for routine lipid level measurement is broadly needless and is improbable to affect patient clinical prospect stratification, while non-fasting lipid measurement might boost patient conformity and safety. Furthermore, UK NICE guidelines have advocated non-fasting lipid testing in the primary prevention setting since $2014 .{ }^{23}$ Requirement of fasting makes sample collection for lipid profile avoidably difficult for all the patients. The fasting state is that used conventionally; however, it would be easier for all the patients if a lipid profile sample could be drawn at any time of the day, irrespective of the timing and the content of the last meal.

\section{Drawbacks of Fasting Samples}

a. Fasting is difficult for children.

b. Fasting is a barrier for population screening.

c. Some people might not be true regarding their food intake/ fasting state.

d. Fasting restricts the use of point-of-care testing.

e. Fasting can be a risky affair for the patient of diabetes as they might end up in hypoglycaemic shock.

f. Patients are often annoyed by return on a separate visit for a fasting lipid profile and may fail on essential testing.

g. Fasting requirements can add to the overall costs of lipid testing.

h. Laboratories are burdened by a large number of patients for fasting lipid profile samples in the morning.

\section{Advantages of Non-Fasting Lipid Profile}

a. The most obvious advantage of non-fasting rather than fasting lipid profile measurements is that it simplifies blood sampling for patients, physicians and testing laboratories. ${ }^{11-15}$

b. Patients who have not fasted do not have to make another appointment to have their blood drawn.

c. Non-fasting specimen collection is more convenient for patients; it promotes patient acquiescence towards lipid profile checking. ${ }^{11-15}$

Among all studies comparing non-fasting with fasting lipid profiles, only minimal changes in lipid profile concentrations were noted which were clinically insignificant. Also, studies have persistently reported that non-fasting lipid profile suffice for screening of cardiovascular disease risk. ${ }^{8-12}$ However, limitations of nonfasting lipid levels include (i) non-fasting levels may be less standardized, (ii) abnormal/normal lipid profile cut-points are not established.

\section{Limitations}

a. Sample size was small.

b. Second sample was not taken at fixed intervals after the last meal (random sample).

c. No diet record of participant before blood sampling. Practically also such information is not available before blood sampling.

d. As superiority of fasting lipid testing was not proven over non-fasting lipid profile, it is justifiable to consider non-fasting lipid testing in persons who present for a routine clinic visit. 


\section{CONCLUSIONS}

Fasting samples are preferable for serum lipid profile measurement in all individuals with serum triglyceride levels greater than $350 \mathrm{mg} / \mathrm{dL}$ (in whom the Friedwald equation for calculating LDL should not be used). But, non-fasting samples for lipid profile can be used for cardiovascular risk determination in the general people as it reduces patient's inconvenience and promotes patient acquiescence towards lipid profile checking.

\section{Recommendations}

a. Fasting is not normally required for determining the plasma lipid profile in the general population.

b. When non-fasting serum triglyceride concentration is very high $(>350 \mathrm{mg} / \mathrm{dL})$, lipid profile may be repeated in fasting state.

\section{ACKNOWLEDGEMENT}

We would like to acknowledge the support from technicians of Central Clinical Biochemistry Laboratory of the tertiary care institute.

\section{REFERENCES}

[1] Nigam PK. Serum lipid profile: fasting or non-fasting? Indian J Clin Biochem 2011;26(1):96-7.

[2] Campos H, Khoo C, Sacks FM. Diurnal and acute pattern of postprandial apolipoprotein B-48 in VLDL, IDL and LDL from normolipidemic humans. Atherosclerosis. 2005;181(2):345-51.

[3] Third report of the National Cholesterol Education Program (NCEP) Expert panel on detection, evaluation and treatment of high blood cholesterol in adults (adult treatment panel III) final report. Circulation 2002;106(25):3143-421.

[4] De Backer G, Ambrosioni E, Borch-Johnson K, et al. European guidelines on cardiovascular disease and prevention in clinical practice. Atherosclerosis 2003;171(1):145-55.

[5] Broder J. Lipid profiles: fasting not necessary, concludes large study. Medscape Nov 12, 2012.

[6] Craig SR, Amin RV, Russell DW, et al. Blood cholesterol screening influence of fasting state on cholesterol results and management decisions. J Gen Intern Med 2000;15(6):395-9.

[7] Wilder LB, Bachorik PS, Finney CA, et al. The effect of fasting status on the determination of low-density and high-density lipoprotein cholesterol. Am J Med 1995;99(4):374-7.

[8] Eberly LE, Stamler J, Neaton JD. Relation of triglyceride levels, fasting and nonfasting, to fatal and nonfatal coronary heart disease. Arch Intern Med 2003;163(9):1077-83.
[9] Bansal S, Buring JE, Rifai N, et al. Fasting compared with nonfasting triglycerides and risk of cardiovascular events in women. JAMA 2007;298(3):309-16.

[10] Nordestgaard BG, Benn M, Schnohr P, et al. Nonfasting triglycerides and risk of myocardial infarction, ischemic heart disease, and death in men and women. JAMA 2007;298(3):299-308.

[11] Langsted A, Freiberg JJ, Nordestgaard BG. Fasting and nonfasting lipid levels: influence of normal food intake on lipids, lipoproteins, apolipoproteins, and cardiovascular risk prediction. Circulation 2008;118(20):2047-56.

[12] Mora S, Rifai N, Buring JE, et al. Fasting compared with nonfasting lipids and apolipoproteins for predicting incident cardiovascular events. Circulation 2008;118(10): 993-1001.

[13] Watts GF, Cohn JS. Whither the lipid profile: feast, famine, or no free lunch? Clin Chem 2011;57(3):363-5.

[14] Gaziano JM. Should we fast before we measure our lipids? Arch Intern Med 2012;172(22):1705-6.

[15] Khera AV, Mora S. Fasting for lipid testing: is it worth the trouble? Arch Intern Med 2012;172(22):1710-2.

[16] Friedewald WT, Levy RI, Fredrickson DS. Estimation of the concentration of low-density- lipoproteins cholesterol in plasma, without use of the preparative ultracentrifuge. Clin Chem 1972;18(6):499-502.

[17]Simundic AM, Cornes M, Grankvist $K$, et al. Standardization of collection requirements for fasting samples: for the Working Group on Preanalytical Phase (WG-PA) of the European Federation of Clinical Chemistry and Laboratory Medicine (EFLM). Clin Chim Acta 2014;432:33-7.

[18] Zilversmit DB. Atherogenic nature of triglycerides, postprandial lipidemia, and triglyceride-rich remnant lipoproteins. Clin Chem 1995;41(1):153-8.

[19] Steiner MJ, Skinner AC, Perrin EM. Fasting might not be necessary before lipid screening: a nationally representative cross-sectional study. Pediatrics 2011;128(3):463-70.

[20] Langsted A, Nordestgaard BG. Nonfasting lipids, lipoproteins, and apolipoproteins in individuals with and without diabetes: 58434 individuals from the Copenhagen General Population Study. Clin Chem 2011;57(3):482-9.

[21] Sidhu D, Naugler C. Fasting time and lipid levels in a community-based population: a cross-sectional study. Arch Intern Med 2012;172(22):1707-10.

[22] Nordestgaard BG, Hilsted L, Stender S. Plasmalipider hos ikkefastende patienter og signalvaerdier på laboratoriesvar. Ugeskrift for Læger 2009;171(13):1093.

[23]NICE clinical guideline CG181. Lipid modification: cardiovascular risk assessment and the modification of blood lipids for the primary and secondary prevention of cardiovascular disease. https://www.nice.org.uk/guidance/cg181/evidence/lipi d- modification-update-full-guideline-243786637 (24 October 2015). 